\title{
Image Reconstruction Using Impedance Measurement as EIT Technique
}

\author{
Munish Kumar ${ }^{1}$, Maninder Kaur ${ }^{2}$ and Dr. K.V.P. Singh ${ }^{3}$ \\ ${ }^{I}$ Doaba Institute of Engg. and Tech., Kharar, Mohali \\ ${ }^{2}$ Assistant Professor DIET, Kharar, Mohali \\ ${ }^{3}$ DIET, Karar, Mohali
}

\begin{abstract}
Electrical Impedance Tomography (EIT) is a radiation-less imaging technology and offers a noninvasive imaging system for human organs. EIT is fast, inexpensive and portable as compared to other imaging techniques. The presented work is based on potential profile computed from the raw data as obtained from the body surface via the electrodes array placed around the organ. A 16 electrode array is proposed for extracting the potential profile. The potential profile is obtained in digital format by sing a 24 bit ADC in order to get a higher resolution potential profile. This helps in reconstructing the organ image at highly resolution.
\end{abstract}

Keywords: EIT

\section{Introduction}

Electrical Impedance Tomography (EIT) is a relatively new medical imaging method, which has powerful potential for providing novel images of brain function. It is fast, portable - about the size of a video recorder and PC, safe and inexpensive, but currently has a relatively poor spatial resolution. It produces images of the internal electrical impedance of a subject using rings of ECG like electrodes on the skin.

Three-dimensional Electrical Impedance Tomography (EIT) is a technique that has the potential to provide estimates of reservoir saturation at multiple scales by determining the resistivity distribution within the subsurface. In theory EIT is well suited for researching geothermal systems due to the large contrast in resistivity between the liquid and vapor phases. Here in our initial laboratory investigation we have applied the EIT technique to measure the saturation distribution within a core. Experiments have indicated EIT is a viable technique for studying the displacement characteristics of fluids with contrasting resistivity, and is capable of detecting displacement fronts in near real-time. The concept of studying the internal properties of a medium by imposing a current, measuring the

\section{Related Works}

Sagar K. Dhar \& Quazi D.Hossain et al (2012) in the paper title "Non-invasive Bio-impedance Measurement Using Voltage-Current Pulse Technique" proposed Noninvasive detection is a fundamental prerequisite for pervasive healthcare system and bio sensing. Along with different non-invasive techniques such as ultrasound, X-ray, magnetic resonance and optical imaging, electrical impedance spectroscopy is emerging as more suitable technique for tissue level diagnosis. This paper presents a noninvasive technique for bioelectrical impedance estimation by means of impedance spectroscopy applying voltage current pulse technique and least square curve fitting method. Simple circuit for current injection and voltage detection is presented with common mode noise suppression circuit requires only two electrodes would reduce problems due to electrode polarization. [1].

David Silvera Tawil, David Rye \& Mari Velonaki et al (2011) in the paper title "Improved Image Reconstruction for an EIT-Based Sensitive Skin With Multiple Internal Electrodes" proposed Electrical impedance tomography (EIT) is a technique used to estimate the internal conductivity of an electrically conductive body by using measurements made only at its boundary. If this body is made of a thin, flexible, and stretchable material that responds to touch with local changes in conductivity, it can be used to create an artificial sensitive skin. Mathematically, the EIT reconstruction problem is an ill-posed nonlinear inverse problem in which it is commonly assumed that electrodes are located only on the surface of the body [2].

Tushar Kanti Bera1, Samir Kumar Biswas, K. Rajan, Nagaraju Jampanal et al (2011) in the paper title "Improving the Image Reconstruction in Electrical Impedance Tomography (EIT) with Block Matrixbased Multiple Regularization (BMMR): A Practical Phantom Study" proposed Conductivity image reconstruction is studied with a Block Matrix based Multiple Regularization (BMMR) technique in Electrical 
Impedance Tomography (EIT) using practical phantoms. [3].

Zhou Zhou, Li Nan, Xu Hui, Guo Jin, Sun Zhaolin, Liu Haijun, Yu Hongqi et al (2011) in the paper title " The Design and Implementation of a Portable EIT Telemedicine System" proposed a portable electrical impedance tomography telemedicine system which supports field diagnosis and electronic medical record building. [4].

Robert W. Stacey et al (2006) in the paper title "Electrical Impedance Tomography" proposed EIT is a viable technique for studying the displacement characteristics of fluids with contrasting resistivity, and is capable of detecting displacement fronts in near real-time. [5].

Olga Mikhailova, Wei-Wen Dai \& Johnny Chee, et al (1996) in the paper title "Pattern Recognition Based Imaging In Electrical Impedance Tomography" proposed Images of electrical conductivity distribution obtained by Electrical Impedance [6].

Hun Wi \& Eung Je Woo et al (1994) in the paper title "Design of KHU Mark2 Multi-frequency EIT System", proposed the design of a new multi-frequency electrical impedance tomography (EIT) system KHU Mark [7].

Eung Je Woo, Ping, John G. Webster et al (1992) in the paper title "Measuring Lung Restivity using EIT" proposed EIT techniques are proposed in the measurement of lung resistivity for detection and monitoring of apnea and edema. [8].

\section{Proposed EIT System}

The system consists of an electrode array which is connected to the body part of interest. Input is given from a constant current source. The electrodes are connected to a multiplexer who gives only one signal out of many at one time. The output of multiplexer is fed to the signal conditioning circuit. This unit basically consists of an amplifier, a filter and a buffer. The signal is amplified because body signals are very weak and of very small magnitude. After amplification, the signal is passed through filter circuit to eliminate higher frequencies and noise signals, if any. Then the analog signal is converted into digital form using $\mathrm{A} / \mathrm{D}$ converter. After digitization, the data is processed using micro controller and saved in memory. The results are displayed using display devices.

\section{Image Reconstruction Module}

Tthe measured values of impedance are used to reconstruct the image of particular body tissues. Different materials have different electrical properties. Due to these differences, the images for unhealthy tissue will be different from a healthy one. The image reconstruction algorithm will be developed using MATLAB environment.

For two dimensional (2D) EIT, there are no substantial differences between these two kinds of methods. However, when three dimensional (3D) EIT is applied, direct methods become deficient in computing time and memory consumption since they are usually based on some kind of matrix factorization. Therefore, iterative methods can be much more efficient for 3D EIT reconstruction. For iterative algorithms, convergence is very important and preconditioning performs as an effective way to enhance the convergence rate of the algorithm.

\section{V. $\quad$ EIT in Medical field-}

It is an exciting possibility to consider making images of the spatial variation of the electrical properties of biological tissue. Biological tissues have complex electrical impedance related to the tissue dimension, the internal structure and the arrangement of the constituent cells. Therefore, the electrical impedance can provide useful information based on heterogeneous tissue structures, physiological states and functions. In addition the concepts of time varying distribution of electrical properties inside a human body such as electrical conductivity and permittivity can be used to analyze a variety of medical conditions. Biological tissue exhibits at least two important passive electrical properties.

a) It comprises free charge carriers and may be considered an electrical conductor. It would be expected that electrical conductivity is a characteristic property of different tissues and that images of electrical conductivity may resolve structure and even be indicative of pathology.

b) Tissue also contains bound charges leading to dielectric effects and it might also be possible to form an image of relative electrical permittivity.

Electrical conductivity is a useful property to obtain images. The conductivity of biological material is linear within $5 \%$ in the region $100 \mathrm{~Hz}$ to $100 \mathrm{kHz}$. High-conductivity materials allow the passage of both direct and alternating currents and high-permittivity materials allow the passage of only alternating currents. Both of 
these properties are of interest in medical systems since different tissues have different conductivities and permittivity's. Electrical properties such as the electrical conductivity $\boldsymbol{\sigma}$ and the electric permittivity $\varepsilon$ determine the behavior of materials under the influence of electric fields. The complex admittivity function $\gamma$ for any material at frequency $\omega$ is given by:-

$$
\gamma(x, \omega)=\sigma(x)+\mathrm{i} \omega \epsilon(x), \quad \text { where } \mathrm{i}=\sqrt{ }-1 .
$$

Different body parts show different electrical properties. Table 1 shows the electrical properties of various biological tissues measured at frequency $10 \mathrm{kHz}$.

A map of $\sigma(x)$ and $\varepsilon(x)$ can be used to infer the structure of internal electrical impedance. This electrical impedance distribution within medium is not measured directly. It is calculated from boundary voltage measurement which is a function of impedance and current. Usually the conducting electrodes are attached to the skin of the subject and small alternating currents are applied to some of the electrodes. The resulting electrical voltages are measured, and the process may be repeated for numerous different configurations of applied current. Using different current injection patterns and voltage measurement sequences, an approximation of the spatial distribution of the impedance or changes in impedance within the object are reconstructed.

\section{Results}

Followings are the images reconstructed from the EIT data as obtained using the data acquisition board based EIT concept. The EIT data are in the form of electrical impedance computed by measuring the voltage across the electrodes placed in an array and mounted on the arm:

\begin{tabular}{|l|l|l|l|l|l|l|l|l|}
\hline Electrode No. & E1 & E2 & E3 & E4 & E5 & E6 & E7 & E8 \\
\hline Fig. 1 & 100 & 115 & 112 & 124 & 110 & 125 & 114 & 121 \\
\hline Fig. 2 & 130 & 135 & 132 & 132 & 131 & 135 & 132 & 130 \\
\hline Fig. 3 & 170 & 170 & 170 & 170 & 170 & 170 & 170 & 170 \\
\hline Fig. 4 & 231 & 232 & 230 & 235 & 232 & 233 & 231 & 231 \\
\hline
\end{tabular}

\begin{tabular}{|l|l|l|l|l|l|l|l|l|}
\hline Electrode No. & E9 & E10 & E11 & E12 & E13 & E14 & E15 & E16 \\
\hline Fig. 1 & 127 & 115 & 113 & 110 & 112 & 115 & 114 & 111 \\
\hline Fig. 2 & 132 & 133 & 131 & 135 & 132 & 131 & 134 & 131 \\
\hline Fig. 3 & 170 & 170 & 170 & 170 & 170 & 170 & 170 & 170 \\
\hline Fig. 4 & 101 & 101 & 101 & 101 & 101 & 101 & 101 & 101 \\
\hline
\end{tabular}

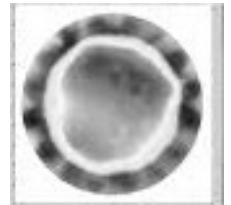

Fig. 1

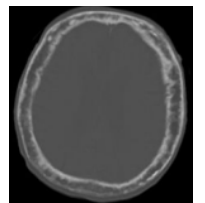

Fig. 2

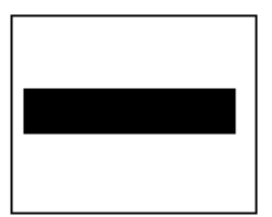

Fig. 3

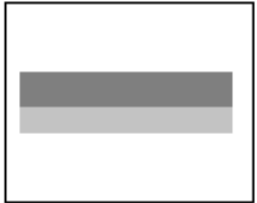

Fig. 4

Fig. 1 and Fig. 2 are taken from the human wrist and fig. 3 and fig. 4 are taken for black color rod and two color reod respectively.

\section{CONCLUSION}

It is observed from the EIT table consisting of data for same organ in the form of 16 electrodes that the impedance varies at a high rate in order to capture the abnormality in the body organ. Also, the impedance is in the order of mega ohms and thereby change in impedance is also in several mega ohms and this gives the ease of observing fair variation between the normal and abnormal condition of an organ. 


\section{References}

[1] Sagar K. Dhar and Quazi D. Hossain, "Non-invasive Bio-impedance Measurement Using Voltage-Current Pulse Technique", International Conference on Electrical, Electronics and Biomedical Engineering (ICEEBE'2012) Penang (Malaysia) May 19-20, 2012

[2] David Silvera Tawil, Student Member, IEEE, David Rye, Member, IEEE, and Mari Velonaki, "Improved Image Reconstruction for an EIT-Based Sensitive Skin With Multiple Internal Electrodes", IEEE Transactions on Robotics, Vol. 27, No. 3, June2011

[3] Tushar Kanti Bera1, Samir Kumar Biswas2, K. Rajan2, Nagaraju Jampana1, "Improving the Image Reconstruction in Electrical Impedance Tomography (EIT) with Block Matrix-based Multiple Regularization (BMMR): A Practical Phantom Study", 978-1-46730126-8/11/\$26.00_c 2011 IEEE

[4] Zhou Zhou, Li Nan, Xu Hui, Guo Jin, Sun Zhaolin, Liu Haijun, Yu Hongqi, "The Design and Implementation of a Portable EIT Telemedicine System", 2011 Second International Conference on Intelligent System Design and Engineering Application

[5] Robert W. Stacey, "Electrical Impedance Tomography", June 2006, Stanford Geothermal Program, Interdisciplinary Research in Engineering and Earth Sciences Stanford University

[6] Olga Mikhailova, Wei-Wen Dai, Johnny Chee, BME Laboratory, "Pattern Recognition Based Imaging In Electrical Impedance Tomography", 18th Annual International Conference of the IEEE Engineering in Medicine and Biology Society, Amsterdam 1996

[7] Hun Wi and Eung Je Woo, "Design of KHU Mark2 Multi-frequency EIT System”, Department of Biomedical Engineering, Kyung Hee University, Yongin, Korea. 1994

[8] Eung Je Woo, Ping, John G. Webster, Measuring Lung Restivity using EIT”, IEEE-1992

\section{Author Profile}

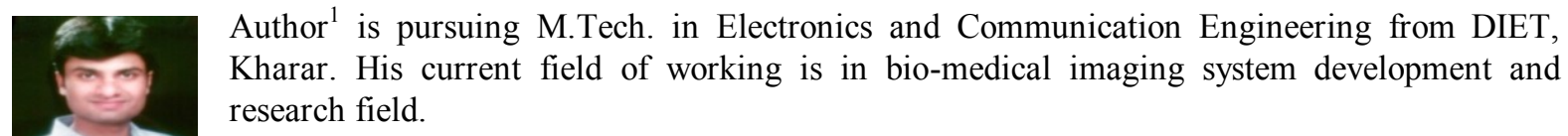

